\title{
Assessment of Liver Function Using Pharmacokinetic Parameters of Gd-EOB-DTPA: Experimental Study in Rat Hepatectomy Model
}

\author{
Myung-Won You $\mathbb{D}^{1,2}$ Hyoung Jung Kim ${ }^{\mathbb{D}},{ }^{1}$ Hyeong-Seok Lim, ${ }^{3}$ So Yeon Kim, ${ }^{1}$ \\ Jae Ho Byun, ${ }^{1}$ Kyung Won Kim, ${ }^{1}$ Dae Wook Hwang, ${ }^{4}$ and Young-Joo Lee ${ }^{4}$ \\ ${ }^{1}$ Department of Radiology and the Research Institute of Radiology, University of Ulsan College of Medicine, \\ Asan Medical Center, Seoul, Republic of Korea \\ ${ }^{2}$ Department of Radiology, Eulji Hospital, Eulji University School of Medicine, Seoul, Republic of Korea \\ ${ }^{3}$ Department of Clinical Pharmacology and Therapeutics, University of Ulsan College of Medicine, \\ Asan Medical Center, Seoul, Republic of Korea \\ ${ }^{4}$ Department of Surgery, University of Ulsan College of Medicine, Asan Medical Center, Seoul, Republic of Korea
}

Correspondence should be addressed to Hyoung Jung Kim; hjk@amc.seoul.kr

Received 5 December 2017; Accepted 12 February 2018; Published 11 March 2018

Academic Editor: Catherine M. Pastor

Copyright (C) 2018 Myung-Won You et al. This is an open access article distributed under the Creative Commons Attribution License, which permits unrestricted use, distribution, and reproduction in any medium, provided the original work is properly cited.

\begin{abstract}
Objectives. To determine whether the pharmacokinetic parameters of Gd-EOB-DTPA can identify the difference in liver function in a rat hepatectomy model. Methods. A total of 56 eight-week-old male Sprague-Dawley rats were divided into the following groups: control group without hepatectomy $(n=16)$, 70\% hepatectomy group $(n=14)$, and $90 \%$ hepatectomy group $(n=$ 26). On postoperative day 2, Gd-EOB-DTPA $(0.1 \mathrm{mmol} / \mathrm{kg})$ was injected intravenously and serial blood samples were obtained. Pharmacokinetic analysis was performed using a noncompartmental method. Statistical analysis was performed using one-way analysis of variance and post hoc pairwise group comparisons. Results. After excluding 6 rats that died unexpectedly, blood samples were obtained from 16, 14, and 20 rats in the control group, $70 \%$ hepatectomy group, and $90 \%$ hepatectomy group. There was a significant increase in area under the concentration-time curve from time zero to the time of the last measurable concentration between the $70 \%$ and $90 \%$ hepatectomy group $(P<0.001)$. The volume of distribution at steady state was significantly decreased between the control and 70\% hepatectomy group $(P<0.001)$. The clearance was significantly different in all pairwise group comparisons $(P<0.001)$. Conclusions. The vascular clearance of Gd-EOB-DTPA can identify the difference in liver function in a rat hepatectomy model.
\end{abstract}

\section{Introduction}

Assessment of liver function is important to determine the prognosis in patients with chronic liver disease, to establish the optimal timing for transplantation or transjugular intrahepatic portosystemic shunt insertion, and to minimize the risk of hepatic failure following major hepatic resection $[1,2]$. In daily clinical practice, serum bilirubin level, serum albumin level, and prothrombin time are methods that are simple and commonly used to assess hepatic function. However, these qualitative liver function tests cannot exactly reflect liver function, because a single measurement of an activity or the concentration of a substance in the blood does not provide data about the volume of distribution, amount of production, or amount of elimination [2]. Thus, when more precise assessments of liver function are required, for example, before major hepatectomy, quantitative liver function tests, such as indocyanine green (ICG), are favored [2].

Hepatic uptake and biliary excretion of ICG involve an organic anion transporter (OAT) system which is increasingly recognized as a major route for the transport of anionic xenobiotics and endogenous substances into the bile [3]. 
The expression of OATs in the liver varies in acute and chronic liver disease and accordingly it is considered to be an important indicator of liver function $[4,5]$. Interestingly, GdEOB-DTPA, which is a widely used contrast agent for liver MR imaging, uses the same OAT as ICG [6-8]. Recent studies used Gd-EOB-DTPA-enhanced liver MR imaging to evaluate liver functions [9-17]. These studies mainly focused on enhancement of the hepatic parenchyma on Gd-EOB-DTPAenhanced liver MR imaging. However, hepatic parenchymal enhancement on Gd-EOB-DTPA-enhanced liver MR is associated with decreased serum concentrations of the contrast agent. Therefore, measurements of serum Gd-EOB-DTPA concentrations can represent a more direct method to assess hepatic function than evaluations of hepatic parenchymal enhancement on liver MR imaging.

The multiple and various functions of the liver preclude an easy single reference standard for assessments of liver function [18]. In the rat hepatectomy model, defined combinations of liver lobes are removed, which are classified based on the approximate relative resected liver mass as 30\%, 50\%, $70 \%$, and $90 \%$ hepatectomy $[19,20]$. A reference standard of liver function based on rat hepatectomy model may represent overall liver functions and hepatectomy with increasing lobe resections is a convenient model to assess liver functions. The purpose of this study was to determine whether pharmacokinetic parameters of Gd-EOB-DTPA could identify the difference in liver function in a rat hepatectomy model.

\section{Materials and Methods}

This study was reviewed and approved by the Institutional Animal Care and Use Committee (IACUC) ${ }^{*}$ BLINDED* $^{*}$ Institute for Life Sciences, ${ }^{*}$ BLINDED* Medical Center. The committee abides by the institute of Laboratory Animal Resources (ILAR) guide.

2.1. Animals. A total of 56 eight-week-old male SpragueDawley (SD) rats (Orientbio Inc., Korea) were used. Several major surgical organizations have established guidelines that recommend that at least $20 \%$ of a normal liver with intact vascular and biliary tree should remain for the surgical resection of liver tumors [21]. Therefore, we divided rats into the following groups: control group without hepatectomy $(n=16), 70 \%$ hepatectomy group $(n=14)$, and $90 \%$ hepatectomy group $(n=26)$. The $70 \%$ hepatectomy group was designed to represent hepatectomy with abnormal but sufficient liver function for survival. The $90 \%$ hepatectomy group was designed to represent hepatectomy with marginal liver function for survival.

2.2. Rat Hepatectomy. Each rat was weighed prior to surgery. Anesthesia and rat hepatectomy were performed by an experienced veterinarian (S.H.H., who had 8 years of experience in animal surgery). For anesthesia, an isoflurane vaporizer (RC2, Vetequip, USA) with an isoflurane concentration of $1 \%-4 \%$ and an oxygen flow rate of $0.5 \mathrm{~L} / \mathrm{min}$ was used. Removal by crude ligation of the wide base of the median lobe and right superior lobe may cause constriction of the vena cava and subsequent functional impairment of the remnant liver [19]. Therefore, we used the clamping and piercing suture technique proposed by Madrahimov et al. [19]. After placing a Mosquito clamp around the base of the each lobe, the liver tissue was dissected just above the clamp. Piercing sutures that penetrated the entire parenchyma were placed below the clamp. Immediately after the operation, animals received $1.5 \mathrm{~mL} 10 \%$ glucose and $1.5 \mathrm{~mL}$ normal saline subcutaneously, along with intramuscular $0.2 \mathrm{~mL}$ Gentamycin (Choongwae, Korea) and 0.2 mL Diclofenac Sodium (Samjin, Korea). Animals had free access to water and feed.

2.3. Blood Sampling. Blood sampling was performed on postoperative day (POD) 2. Each rat was weighed prior to blood sampling. Femoral artery cannulation was performed for blood sampling. Gd-EOB-DTPA (Primovist; Bayer HealthCare, Berlin, Germany) ( $0.1 \mathrm{mmol}$ gadolinium per $\mathrm{kg}$ body weight, $0.4 \mathrm{~mL} / \mathrm{kg}$ ) was used for pharmacokinetic analyses. The Gd-EOB-DTPA was diluted 15-fold using normal saline and injected into the tail vein. Blood samples of $120 \mu \mathrm{L}$ were obtained $1,3,5,10,20,30,60$, and 90 min after injecting GdEOB-DTPA. For each $120 \mu \mathrm{L}$ of blood, $50 \mu \mathrm{L}$ was used for gadolinium measurement and $70 \mu \mathrm{L}$ was reserved.

2.4. Measurement of Liver Weight. All animals were sacrificed using a $\mathrm{CO}_{2}$ chamber after the completion of blood sampling. In each group, the wet liver weight was measured after explantation. The total wet liver weight was measured after removing the inferior vena cava and portal vein. To determine the absolute weight of each liver lobe, the weight of each liver lobe was also measured after dissecting each lobe with the same technique for rat hepatectomy. The relative weight of each lobe was calculated using the following formula: relative weight of lobe $(\%)=$ weight of lobe $(g) /$ total liver weight $(g)$.

2.5. Gadolinium Measurements and Pharmacokinetic Anal$y$ sis. Blood samples were assayed for gadolinium using an inductively coupled plasma-mass spectrometry (ICP-MS). Blood samples were centrifuged to collect all material at the bottom of the caps. We added $50 \mu \mathrm{L}$ internal standard (100 nM Terbium-nitrate). The mixture was dried at $90^{\circ} \mathrm{C}$. Then, $20 \mu \mathrm{l} 30 \%$ hydrogen peroxide and $50 \mu \mathrm{L}$ concentrated nitric acid were added and the mixture was heated to $120^{\circ} \mathrm{C}$ for about $30 \mathrm{~min}$ at increased pressure. After cooling, the volume was made up to $1 \mathrm{~mL}$ with water. The solution was further diluted depending on the expected gadolinium concentration in the samples. Final dilutions were injected to the ICPMS to obtain the concentration measurements. The ICP-MS (Agilent 7900) was calibrated using dilutions of commercial, certified standards (Merck) with $0,1,10$, and $100 \mathrm{nmol} \mathrm{Gd/L}$ and $10 \mathrm{nM} \mathrm{Tb}$. All sample measurements were made within the calibrated range. Blank digestions (empty caps) were also included in the procedure and resulted in Gd concentrations well below the limit of quantification. Using our new ICPMS, the limit of quantification was about $0.1 \mathrm{nmol} \mathrm{Gd} / \mathrm{L}$. The upper limit of the linear measurement range was about $20 \mu \mathrm{mol} \mathrm{Gd} / \mathrm{L}$. Serial serum concentration-time profiles of 
TABLE 1: The body weight and explanted liver weight in each study group of SD rats.

\begin{tabular}{lccc}
\hline & $\begin{array}{c}\text { Control } \\
(n=16)\end{array}$ & $\begin{array}{c}70 \% \\
\text { hepatectomy } \\
(n=14)\end{array}$ & $\begin{array}{c}90 \% \\
\text { hepatectomy } \\
(n=20)\end{array}$ \\
\hline Preoperative body weight $(\mathrm{g})$ & $303.69 \pm 24.52$ & $287.14 \pm 13.82$ & $296.00 \pm 23.86$ \\
Postoperative body weight & $\mathrm{NA}$ & $268.21 \pm 15.36$ & $262.55 \pm 21.23$ \\
Explanted liver weight $(\mathrm{g})$ & $12.87 \pm 1.70$ & $8.49 \pm 0.90$ & $4.82 \pm 0.70$ \\
\hline
\end{tabular}

NA: not available.

gadolinium for each rat were analyzed using a noncompartmental method with the WinNonlin 6.3 (Pharsight Corporation, Mountain View, CA, USA). All analyses were made based on actual times of sampling. Pharmacokinetic analysis was performed using a noncompartmental method [22, 23]. The individual area under the concentration curve (AUC) from time zero to time of last measurable concentration was indicated as $\mathrm{AUC}_{\mathrm{last}}$ and $\mathrm{AUC} *$ time versus time curve from time zero to time of last measurable concentration was indicated as $\mathrm{AUMC}_{\text {last }}$. These $\mathrm{AUC}_{\text {last }}, \mathrm{AUMC}_{\text {last }}$ and time product were estimated by linear trapezoidal summation in the ascending period and by log/linear trapezoidal summation in the descending period. The AUC from time zero extrapolated to infinite time $\left(\mathrm{AUC}_{\text {inf }}\right)$ and the AUMC from time zero extrapolated to infinite time $\left(\mathrm{AUMC}_{\text {inf }}\right)$ were calculated as the sum of the $\mathrm{AUC}_{\text {last }}$ and $C_{\text {last }} / \lambda_{\mathrm{z}}\left(\mathrm{AUC}_{\text {extrapolated }}\right)$ and as the sum of $\mathrm{AUMC}_{\text {last }}$ and $C_{\text {last }} *$ time $/ \lambda^{*}{ }_{\mathrm{z}}\left(\mathrm{AUMC} \mathrm{C}_{\text {extrapolated }}\right)$, respectively, in which $C_{\text {last }}$ corresponds to the last predicted concentration. $\lambda_{\mathrm{z}}$ and $\lambda^{*}{ }_{\mathrm{z}}$ are the rate constant of the terminal phase calculated by linear regression of the slope of the terminal portion of the log-transformed serum concentration versus time curve and serum concentration $*$ time versus time curve, respectively. Clearance (CL) was computed as the dose $/ \mathrm{AUC}_{\mathrm{inf}} \cdot V_{\mathrm{z}}$ was volume of distribution during terminal phase $\left(C L / \lambda_{z}\right.$, terminal phase refers to post distribution or elimination phase), and $V_{\text {ss }}$ was volume of distribution at steady state (dose * (AUMC/AUC $\left.{ }^{2}\right)$, steady state refers to distribution phase at free concentration in plasma being equal to the free concentration in the tissue). The mean residence time (MRT) was calculated as AUMC/AUC. The terminal elimination half-life $\left(t_{1 / 2 \beta}\right)$ was calculated for each subject as $\ln (2) / \lambda_{z}$, and the effective half-life $\left(t_{1 / 2, \text { eff }}\right)$ was calculated as $0.693 *$ MRT.

2.6. Statistical Analysis. Data were expressed as means \pm standard deviation. For comparisons of the three groups, continuous variables were compared using oneway analysis of variance. A $P$ value of less than 0.050 was considered to indicate a significant difference. When data indicated the presence of significant difference between the three groups, post hoc pairwise group comparisons were made using Student's $t$ test with the Bonferroni correction. Spearman's correlation test was performed and a scatter plot was used to show the correlation between CL and three groups. All analyses were performed using SPSS 21 (SPSS Inc., Chicago, IL, USA).

\section{Results}

3.1. Subjects. The control group consisted of $16 \mathrm{SD}$ male rats and blood sampling was possible in all 16 animals. The $70 \%$ hepatectomy group consisted of 14 SD male rats and blood sampling was possible in all 14 animals. The $90 \%$ hepatectomy group consisted of $26 \mathrm{SD}$ male rats and blood sampling was possible in 20 animals. Two rats died during hepatectomy as a consequence of hemorrhage and four rats died during the postoperative period. Finally, overall 50 rats were included in the pharmacokinetic analysis.

3.2. Liver Weight. Pre- and postoperative body weights and the explanted liver weights in each group are summarized in Table 1 . In the control group $(n=16)$, the weight of total liver was $12.87 \pm 1.70 \mathrm{~g}$, left lateral lobe was $3.98 \pm 0.58 \mathrm{~g}$, median lobe was $4.26 \pm 0.68 \mathrm{~g}$, right lobe was $2.23 \pm 0.37 \mathrm{~g}$, caudate lobe was $0.82 \pm 0.19 \mathrm{~g}$, and paracaval portion was $1.42 \pm 0.49 \mathrm{~g}$. The relative weights were as follows: left lateral lobe, $30.9 \%$; median lobe, $33.1 \%$; right lobe, $17.4 \%$; caudate, $6.4 \%$; and paracaval portion, $11.0 \%$. The sum of the relative weight of the left lateral and median lobe was $64.1 \%$. The sum of the relative weight of the left lateral, median, and right lobe was $81.4 \%$. In the $70 \%$ hepatectomy group $(n=14)$, the weight of the remnant liver at POD 2 was $8.49 \pm 0.90 \mathrm{~g}$. In the $90 \%$ hepatectomy group $(n=20)$, the weight of the remnant liver at POD 2 was $4.82 \pm 0.70 \mathrm{~g}$.

3.3. Pharmacokinetics of $G d-E O B-D T P A$. A summary of the Gd-EOB-DTPA serum concentration-time profiles for the three groups is shown in Figure 1. Pharmacokinetic parameters obtained from these data are summarized in Table 2. Results of overall and pairwise group comparisons of the three groups are summarized in Table 3. Serum Gd-EOBDTPA concentrations declined rapidly during the first $10 \mathrm{~min}$ and then slowly declined until 90 min after injection in the control group (Figure 1), indicating nonlinear pharmacokinetics. This initial rapid reduction in serum Gd-EOB-DTPA concentrations was similar in the $70 \%$ and $90 \%$ hepatectomy group. In pairwise group comparisons, all pharmacokinetics parameters showed significant difference between the $70 \%$ and $90 \%$ hepatectomy group and between control and $90 \%$ hepatectomy group. Only CL, $V_{\mathrm{ss}}$, and $V_{\mathrm{z}}$ showed significant decrease between the control and 70\% hepatectomy group. The CL, $V_{\mathrm{ss}}$, and $V_{\mathrm{z}}$ were significantly different in all pairwise group comparisons. Scatter plots showing a correlation of CL 
TABLE 2: The summary of pharmacokinetic parameters of Gd-EOB-DTPA.

\begin{tabular}{lccc}
\hline & $\begin{array}{c}\text { Control } \\
(n=16)\end{array}$ & $\begin{array}{c}70 \% \\
\text { hepatectomy } \\
(n=14)\end{array}$ & $\begin{array}{c}90 \% \text { hepatectomy } \\
(n=20)\end{array}$ \\
\hline $\mathrm{AUC}_{\text {last }}(\mu \mathrm{mol} \cdot \mathrm{min} / \mathrm{L})$ & $2501.0 \pm 922.6$ & $3956.6 \pm 1067.2$ & $9234.5 \pm 3891.5$ \\
$\mathrm{AUC}_{\text {inf }}(\mu \mathrm{mol} \cdot \mathrm{min} / \mathrm{L})$ & $2659.6 \pm 1099.2$ & $4292.3 \pm 1281.8$ & $14199.8 \pm 10949.0$ \\
$\mathrm{AUC}_{\text {extrapolated }}(\%)$ & $5.1 \pm 3.2$ & $7.1 \pm 3.3$ & $24.7 \pm 15.2$ \\
$\mathrm{AUC}_{\text {inf }} / \mathrm{dose}(\mu \mathrm{mol} \cdot \mathrm{min} / \mathrm{L} / \mu \mathrm{mol})$ & $90.1 \pm 43.1$ & $160.6 \pm 49.0$ & $545.0 \pm 417.5$ \\
$C_{\max }(\mu \mathrm{mol} / \mathrm{L})$ & $238.1 \pm 62.4$ & $264.1 \pm 58.2$ & $334.9 \pm 65.6$ \\
$C_{\max } / \mathrm{Dose}(\mu \mathrm{mol} / \mathrm{L} / \mu \mathrm{mol})$ & $8.0 \pm 2.6$ & $9.9 \pm 2.3$ & $12.9 \pm 2.9$ \\
$\mathrm{CL}(\mathrm{ml} / \mathrm{min})$ & $12.7 \pm 4.1$ & $6.8 \pm 2.3$ & $2.7 \pm 1.5$ \\
$V_{\mathrm{ss}}(\mathrm{mL})$ & $269.7 \pm 70.1$ & $189.1 \pm 36.0$ & $142.1 \pm 39.3$ \\
$V_{\mathrm{z}}(\mathrm{mL})$ & $487.7 \pm 157.0$ & $254.9 \pm 62.1$ & $160.6 \pm 55.2$ \\
$t_{1 / 2 \beta}(\mathrm{min})$ & $27.3 \pm 5.9$ & $27.0 \pm 5.3$ & $52.6 \pm 30.2$ \\
$t_{1 / 2, \mathrm{efff}}(\mathrm{min})$ & $15.6 \pm 4.5$ & $20.3 \pm 4.5$ & $48.1 \pm 30.1$ \\
\hline
\end{tabular}

Data are presented as means \pm standard deviation. $\mathrm{AUC}_{\text {last }}$ : area under the serum concentration-time curve from time zero to time of last measurable concentration; $\mathrm{AUC}_{\text {inf }}$ : $\mathrm{AUC}$ from time zero to time extrapolated to infinite time; $\mathrm{AUC}_{\text {extrapolated }}(\%)$ : $\mathrm{AUC}$ from time of last measurable concentration extrapolated time to infinite time $/ \mathrm{AUC}_{\mathrm{inf}} ; C_{\mathrm{max}}$ : measured peak plasma concentration; CL: clearance; $V_{\mathrm{ss}}$ : steady-state volume of distribution; $V_{\mathrm{z}}$ : terminal phase volume of distribution; $t_{1 / 2 \beta}$ : terminal half-life; $t_{1 / 2, \text { eff }}$ : effective half-life.

TABLE 3: The effects of hepatic resection on pharmacokinetic parameters of Gd-EOB-DTPA.

\begin{tabular}{|c|c|c|c|c|}
\hline & \multirow{2}{*}{$\begin{array}{c}\text { Overall } \\
\text { comparison }^{*}\end{array}$} & \multicolumn{3}{|c|}{$\begin{array}{l}\text { Pairwise group } \\
\text { comparison }^{\dagger}\end{array}$} \\
\hline & & $\begin{array}{c}\text { Control } \\
\text { versus } 70 \% \mathrm{H}\end{array}$ & $\begin{array}{c}70 \% \mathrm{H} \\
\text { versus } 90 \% \mathrm{H}\end{array}$ & $\begin{array}{c}\text { Control } \\
\text { versus } \\
90 \% \mathrm{H}\end{array}$ \\
\hline $\mathrm{AUC}_{\text {last }}(\mu \mathrm{mol} \cdot \mathrm{min} / \mathrm{L})$ & $<.001$ & .394 & $<.001$ & $<.001$ \\
\hline $\mathrm{AUC}_{\text {inf }}(\mu \mathrm{mol} \cdot \mathrm{min} / \mathrm{L})$ & .019 & 1.000 & .001 & $<.001$ \\
\hline $\mathrm{AUC}_{\text {extrapolated }}(\%)$ & $<.001$ & 1.000 & $<.001$ & $<.001$ \\
\hline $\mathrm{AUC}_{\text {inf }} /$ Dose $(\mu \mathrm{mol} \cdot \mathrm{min} / \mathrm{L} / \mu \mathrm{mol})$ & $<.001$ & 1.000 & $<.001$ & $<.001$ \\
\hline$C_{\max }(\mu \mathrm{mol} / \mathrm{L})$ & $<.001$ & .785 & .007 & $<.001$ \\
\hline$C_{\max } /$ Dose $(\mu \mathrm{mol} / \mathrm{L} / \mu \mathrm{mol})$ & $<.001$ & .172 & .007 & $<.001$ \\
\hline $\mathrm{CL}(\mathrm{ml} / \mathrm{min})$ & $<.001$ & $<.001$ & $<.001$ & $<.001$ \\
\hline$V_{\mathrm{ss}}(\mathrm{mL})$ & $<.001$ & $<.001$ & .031 & $<.001$ \\
\hline$V_{\mathrm{z}}(\mathrm{mL})$ & $<.001$ & $<.001$ & .030 & $<.001$ \\
\hline$t_{1 / 2 \beta}(\min )$ & $<.001$ & 1.000 & .002 & .001 \\
\hline$t_{1 / 2, \mathrm{eff}}(\mathrm{min})$ & $<.001$ & 1.000 & $<.001$ & $<.001$ \\
\hline
\end{tabular}

${ }^{*}$ One-way analysis of variance; ${ }^{\dagger}$ post hoc pairwise group comparisons by using Student's $t$ test with Bonferroni correction; H: hepatectomy.

for the three groups had a significant negative correlation $(r=-0.876, P<0.001)$ (Figure 2).

\section{Discussion}

Our present study shows that measurements of serum GdEOB-DTPA concentrations can identify the difference in liver function in a rat hepatectomy model. Among pharmacokinetic parameters, there was a significant increase in the AUC parameters between $70 \%$ and $90 \%$ hepatectomy group. $V_{\text {ss }}$ was significantly decreased between the control and $70 \%$ hepatectomy group. The CL was significantly different in all pairwise group comparisons.
The volume of distribution parameters $\left(V_{\mathrm{ss}}\right.$ and $\left.V_{\mathrm{z}}\right)$ revealed a significant decrease between the control and $70 \%$ hepatectomy group, and they showed a marginally significant difference between $70 \%$ and $90 \%$ hepatectomy group. $V_{\text {ss }}$ and $V_{\mathrm{z}}$ represented the apparent volume of distribution of Gd-EOB-DTPA during steady state and terminal phase. The difference in liver volume was greater between the control and $70 \%$ hepatectomy group than those between the $70 \%$ and $90 \%$ hepatectomy group, which may explain the difference in $V_{\mathrm{ss}}$ and $V_{\mathrm{z}}$ among the three groups. Interestingly, the difference in explanted liver weight between the control and $70 \%$ hepatectomy group was similar to that between the $70 \%$ and $90 \%$ hepatectomy group. The explanted liver weight of the $70 \%$ or $90 \%$ hepatectomy group was not the 


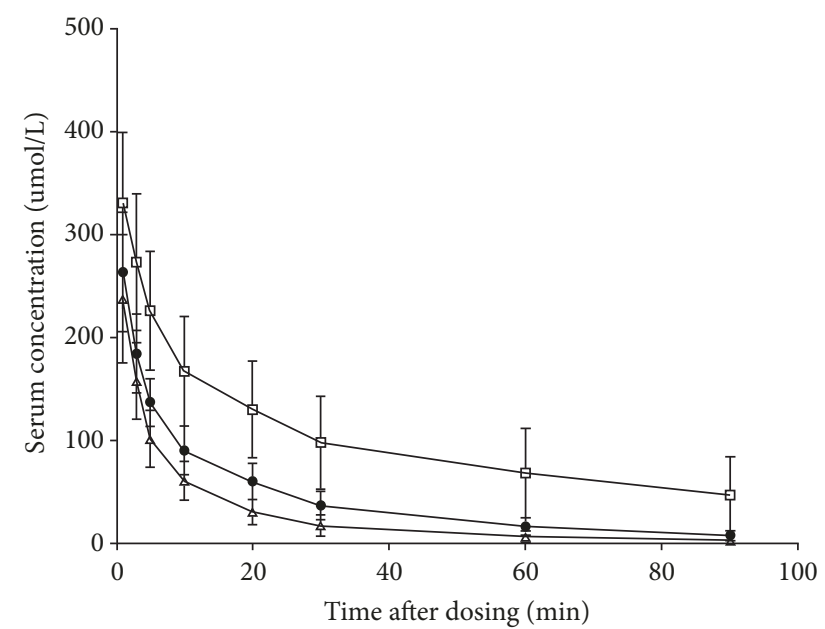

FIGURE 1: Mean serum concentration-time profiles of Gd-EOBDTPA in the control group $(\triangle), 70 \%$ hepatectomy group $(\bullet)$, and $90 \%$ hepatectomy group ( $\square)$. The error bars indicate the standard deviation.

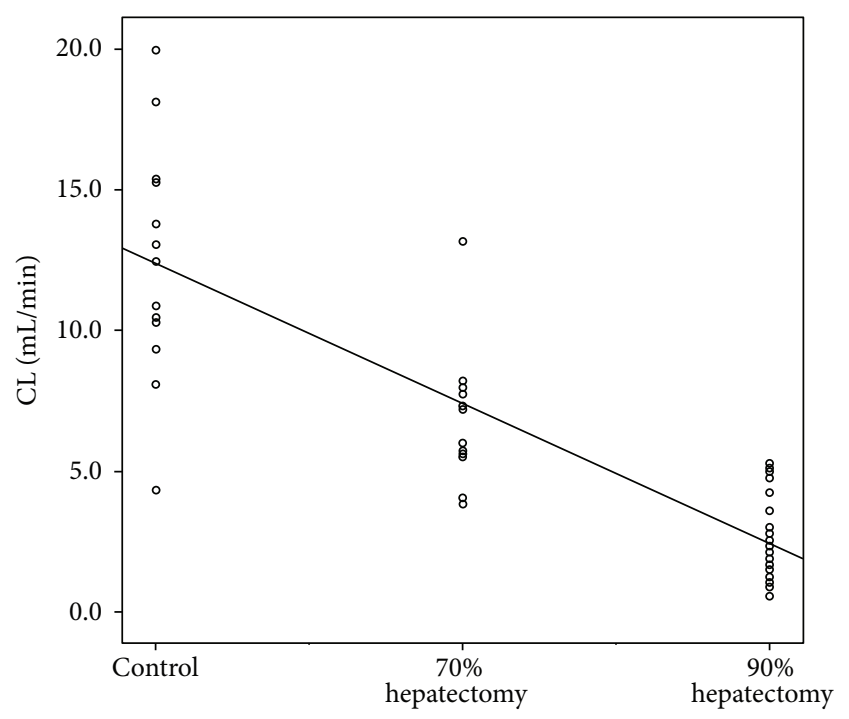

Figure 2: Clearance (CL) of Gd-EOB-DTPA as a function of the control group, $70 \%$ hepatectomy group, and 90\% hepatectomy group showing significant decrease of parameter values with increasing extent of rat hepatectomy $(r=-0.876, P<0.001)$.

normal liver weight but the regenerated liver weight. In our study, the regeneration rate of $70 \%$ and $90 \%$ hepatectomy group was about $65 \%$ and $37 \%$ at POD 2, similar to those of $70 \%$ and $90 \%$ hepatectomy group $(70 \%$ and $40 \%)$ in the previous literatures $[24,25]$. In daily clinical practice, the calculated regenerated liver volume following portal vein embolization does not always correlate with liver function [18]. Therefore, differences in $V_{\mathrm{ss}}$ and $V_{\mathrm{z}}$ between the three groups might represent the differences in liver volume caused by rat hepatectomy rather than the absolute weight of the regenerated liver at POD 2.

On the other hand, the AUC and its related parameters were significantly increased between the $70 \%$ and $90 \%$ hepatectomy group but not between the control and 70\% hepatectomy group. The $\mathrm{AUC}_{\text {last }}$ and $\mathrm{AUC}_{\text {inf }}$ are important pharmacokinetic parameters for noncompartmental analysis as they are frequently used to determine other pharmacokinetic parameters. These results indicate that a critical point in the pharmacokinetics of Gd-EOB-DTPA may present between the $70 \%$ and $90 \%$ hepatectomy. That is, liver with $30 \%$ remnant volume may be sufficient for survival while liver with $10 \%$ remnant volume may be marginal for survival. Our rat hepatectomy model is very useful to verify such findings.

The CL can be defined as a measurement of the volume of plasma that is completely cleared from a substance per unit of time [26]. CL is one of the primary, independent pharmacokinetic parameters. As the total body CL of GdEOB-DTPA will be equal to the hepatic CL plus renal CL, a significant difference in total body CL may result from difference in liver function in a rat hepatectomy models.

Our present study demonstrated that direct measurements of the serum Gd-EOB-DTPA concentrations may represent a novel quantitative liver function test. Among various pharmacokinetic variables, CL showed significant differences in all pairwise group comparisons. However, clinical studies with a large patient cohort will be required to determine whether CL of Gd-EOB-DTPA could represent a new quantitative liver function test. If blood sampling can be performed during Gd-EOB-DTPA-enhanced liver MR imaging, prerequisite data prior to liver surgery, such as liver function, volume, and anatomy, may be obtained simultaneously.

There were several limitations to our present study. First, we did not assess the urinary excretion of Gd-EOBDTPA. Urinary bladder catheterization in male SD rat was impossible because of the long and tortuous course of the urethra. Second, the dosage of Gd-EOB-DPTA used in our study $(0.1 \mathrm{mmol} / \mathrm{kg})$ is 4 -fold greater than the dosage used in human $(0.025 \mathrm{mmol} / \mathrm{kg})$. We used a high dose of Gd-EOBDTPA to compensate much faster metabolic rate of SD rat than human [27]. A previous study also showed that serum concentration-time profile for a dose of $0.05 \mathrm{mmol} / \mathrm{kg}$ was similar to that of $0.5 \mathrm{mmol} / \mathrm{kg}$ in an experiment that used rats [28]. Third, the number of SD rats in each group was different. Initially, the expected number of SD rats without unwanted events in each group was 12 . However, exclusion of the SD rats with unwanted events, such as hemorrhage, inflammation, or various injection sites other than the tail vein, may cause the selection bias. So, we determined to include all SD rats in which successful blood sampling was achieved to prevent the bias introduced by the arbitrary selection of cases.

In conclusion, measurements of serum Gd-EOB-DTPA concentrations can identify the significant difference in liver function in a rat hepatectomy model. Among the various pharmacokinetic parameters, CL of Gd-EOB-DTPA would be the most appropriate parameter to identify the difference in liver function and might have utility as a novel quantitative liver function test.

\section{Abbreviations}

SD: Sprague-Dawley

AUC: Area under the concentration-time curve 
$C_{\text {max }}:$ Measured peak plasma concentration

CL: Clearance

$V_{\text {ss }}$ : Steady-state volume of distribution

$V_{\mathrm{z}}: \quad$ Terminal volume of distribution

$t_{1 / 2 \beta}$ : Terminal half-life

$t_{1 / 2 \text {,eff }}$ : Effective half-life

POD: Postoperative day.

\section{Additional Points}

Key Points. Pharmacokinetic analyses of Gd-EOB-DTPA serum concentrations might have utility as a liver function test. $\mathrm{AUC}_{\text {last }}$ of Gd-EOB-DTPA showed significant increase between $70 \%$ and $90 \%$ hepatectomy group. $V_{\text {ss }}$ showed significant decrease between control and $70 \%$ hepatectomy group. Clearance showed negative correlation with an increasing extent of rat hepatectomy.

\section{Conflicts of Interest}

The authors declare that there are no conflicts of interest regarding the publication of this paper.

\section{Acknowledgments}

This study was supported by grants from Bayer Korea Ltd. Gadolinium concentration analysis was supported by MR and CT Contrast Media Research Team in Bayer Healthcare (SOP ID; BSP-SOP-040). The authors thank Seung-Ho Heo, Asan Institute for Life Sciences, University of Ulsan College of Medicine, Seoul, Korea, for technical support in rat hepatectomy.

\section{References}

[1] A. Zipprich, O. Kuss, S. Rogowski et al., "Incorporating indocyanin green clearance into the Model for End Stage Liver Disease (MELD-ICG) improves prognostic accuracy in intermediate to advanced cirrhosis," Gut, vol. 59, no. 7, pp. $963-$ $968,2010$.

[2] H. Imamura, K. Sano, Y. Sugawara, N. Kokudo, and M. Makuuchi, "Assessment of hepatic reserve for indication of hepatic resection: Decision tree incorporating indocyanine green test," Journal of Hepato-Biliary-Pancreatic Sciences, vol. 12, no. 1, pp. 16-22, 2005.

[3] Y. Shitara, K. Maeda, K. Ikejiri, K. Yoshida, T. Horie, and Y. Sugiyama, "Clinical significance of organic anion transporting polypeptides (OATPs) in drug disposition: Their roles in hepatic clearance and intestinal absorption," Biopharmaceutics \& Drug Disposition, vol. 34, no. 1, pp. 45-78, 2013.

[4] K. M. Giacomini, S.-M. Huang, D. J. Tweedie et al., "Membrane transporters in drug development," Nature Reviews Drug Discovery, vol. 9, no. 3, pp. 215-236, 2010.

[5] X. Gu and J. E. Manautou, "Regulation of hepatic ABCC transporters by xenobiotics and in disease states," Drug Metabolism Reviews, vol. 42, no. 3, pp. 482-538, 2010.

[6] M. Lagadec, S. Doblas, C. Giraudeau et al., "Advanced fibrosis: Correlation between pharmacokinetic parameters at dynamic gadoxetate-enhanced MR imaging and hepatocyte organic anion transporter expression in rat liver," Radiology, vol. 274, no. 2, pp. 379-386, 2015.

[7] P. J. Meier, U. Eckhardt, A. Schroeder, B. Hagenbuch, and B. Stieger, "Substrate specificity of sinusoidal bile acid and organic anion uptake systems in rat and human liver," Hepatology, vol. 26, no. 6, pp. 1667-1677, 1997.

[8] G. Schuhmann-Giampieri, H. Schmitt-Willich, W.-R. Press, C. Negishi, H.-J. Weinmann, and U. Speck, "Preclinical evaluation of Gd-EOB-DTPA as a contrast agent in MR imaging of the hepatobiliary system," Radiology, vol. 183, no. 1, pp. 59-64, 1992.

[9] K. Saito, J. Ledsam, S. Sourbron et al., "Assessing liver function using dynamic Gd-EOB-DTPA-enhanced MRI with a standard 5-phase imaging protocol," Journal of Magnetic Resonance Imaging, vol. 37, no. 5, pp. 1109-1114, 2013.

[10] T. Utsunomiya, M. Shimada, J. Hanaoka et al., "Possible utility of MRI using Gd-EOB-DTPA for estimating liver functional reserve," Journal of Gastroenterology, vol. 47, no. 4, pp. 470-476, 2012.

[11] S. Goshima, M. Kanematsu, H. Watanabe et al., "Gd-EOBDTPA-enhanced MR imaging: Prediction of hepatic fibrosis stages using liver contrast enhancement index and liver-tospleen volumetric ratio," Journal of Magnetic Resonance Imaging, vol. 36, no. 5, pp. 1148-1153, 2012.

[12] B. B. Chen, C. Y. Hsu, C. W. Yu et al., "Dynamic contrastenhanced magnetic resonance imaging with Gd-EOB-DTPA for the evaluation of liver fibrosis in chronic hepatitis patients," European Radiology, vol. 22, no. 1, pp. 171-180, 2012.

[13] A. Yamada, T. Hara, F. Li et al., "Quantitative evaluation of liver function with use of gadoxetate disodium-enhanced MR imaging," Radiology, vol. 260, no. 3, pp. 727-733, 2011.

[14] H. Watanabe, M. Kanematsu, S. Goshima et al., "Staging hepatic fibrosis: Comparison of gadoxetate disodium-enhanced and diffusion-weighted mr imaging - Preliminary observations," Radiology, vol. 259, no. 1, pp. 142-150, 2011.

[15] U. Motosugi, T. Ichikawa, M. Oguri et al., "Staging liver fibrosis by using liver-enhancement ratio of gadoxetic acid-enhanced MR imaging: Comparison with aspartate aminotransferase-toplatelet ratio index," Magnetic Resonance Imaging, vol. 29, no. 8, pp. 1047-1052, 2011.

[16] T. Katsube, M. Okada, S. Kumano et al., "Estimation of liver function using T1 mapping on Gd-EOB-DTPA-enhanced magnetic resonance imaging," Investigative Radiology, vol. 46, no. 4, pp. 277-283, 2011.

[17] U. Motosugi, T. Ichikawa, H. Sou et al., "Liver parenchymal enhancement of hepatocyte-phase images in Gd-EOB-DTPAenhanced MR imaging: which biological markers of the liver function affect the enhancement?" Journal of Magnetic Resonance Imaging, vol. 30, no. 5, pp. 1042-1046, 2009.

[18] C. Cha, "Accessment of hepatic function: implications for the surgical patient," in Blumgart's surgery of the liver, biliary tract and pancreas, W. R. Jarnagin, Ed., pp. 58-64, Elsevier Saunders, Philadelphia, Penn, USA, 5th edition, 2012.

[19] N. Madrahimov, O. Dirsch, C. Broelsch, and U. Dahmen, "Marginal hepatectomy in the rat: From anatomy to surgery," Annals of Surgery, vol. 244, no. 1, pp. 89-98, 2006.

[20] J. Gaub and J. Iversen, "Rat liver regeneration after $90 \%$ partial hepatectomy," Hepatology, vol. 4, no. 5, pp. 902-904, 1984.

[21] J. N. Vauthey, M. A. Choti, and W. S. Helton, "AHPBA/SSO/ SSAT consensus conference on hepatic colorectal metastases: Rationale and overview of the conference," Annals of Surgical Oncology, vol. 13, no. 10, pp. 1259-1260, 2006. 
[22] J. Gabrielsson and D. Weiner, "Non-compartmental Analysis," in Computational Toxicology, vol. 929 of Methods in Molecular Biology, pp. 377-389, Humana Press, Totowa, NJ, 2012.

[23] M. Rowland and TN. Tozer, Clinical pharmacokinetics and pharmacodynamics: concepts and applications, Lippincott Williams and Wilkins, Philadalphia. Penn, USA, 4th edition, 2011.

[24] C. Uribe-Cruz, C. O. Kieling, M. L. López et al., "Encapsulated whole bone marrow cells improve survival in wistar rats after 90\% partial hepatectomy," Stem Cells International, vol. 2016, Article ID 4831524, 9 pages, 2016.

[25] K. J. Andersen, A. R. Knudsen, A.-S. Kannerup et al., "The natural history of liver regeneration in rats: Description of an animal model for liver regeneration studies," International Journal of Surgery, vol. 11, no. 9, pp. 903-908, 2013.

[26] L. Z. Benet and P. Zia-Amirhosseini, "Basic principles of pharmacokinetics," Toxicologic Pathology, vol. 23, no. 2, pp. 115123, 1995.

[27] M. A. Holliday, D. Potter, A. Jarrah, and S. Bearg, "The relation of metabolic rate to body weight and organ size.," Pediatric Research, vol. 1, no. 3, pp. 185-195, 1967.

[28] G. Schuhmann-Giampieri, T. Frenzel, and H. Schmitt-Willich, "Pharmacokinetics in rats, dogs and monkeys of a gadolinium chelate used as a liver-specific contrast agent for magnetic resonance imaging," Arzneimittel-Forschung/Drug Research, vol. 43, no. 8, pp. 927-931, 1993. 


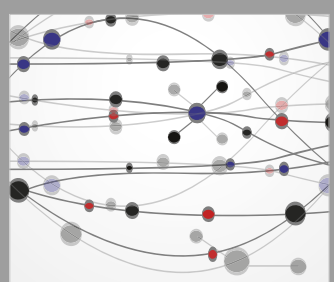

The Scientific World Journal
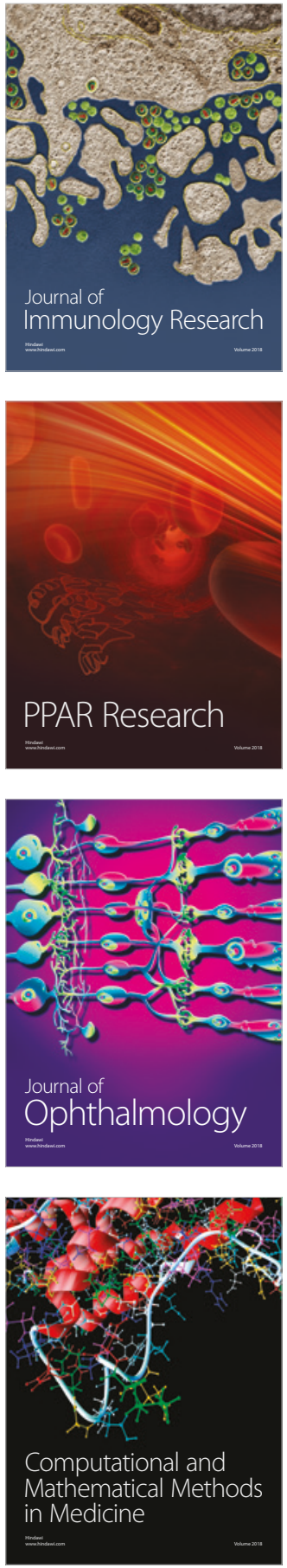

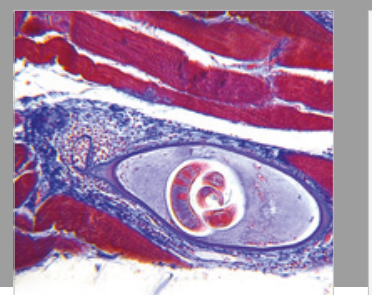

Gastroenterology Research and Practice

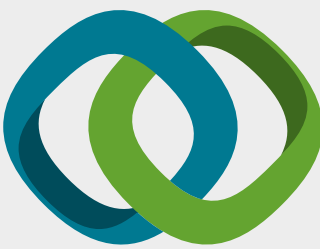

\section{Hindawi}

Submit your manuscripts at

www.hindawi.com
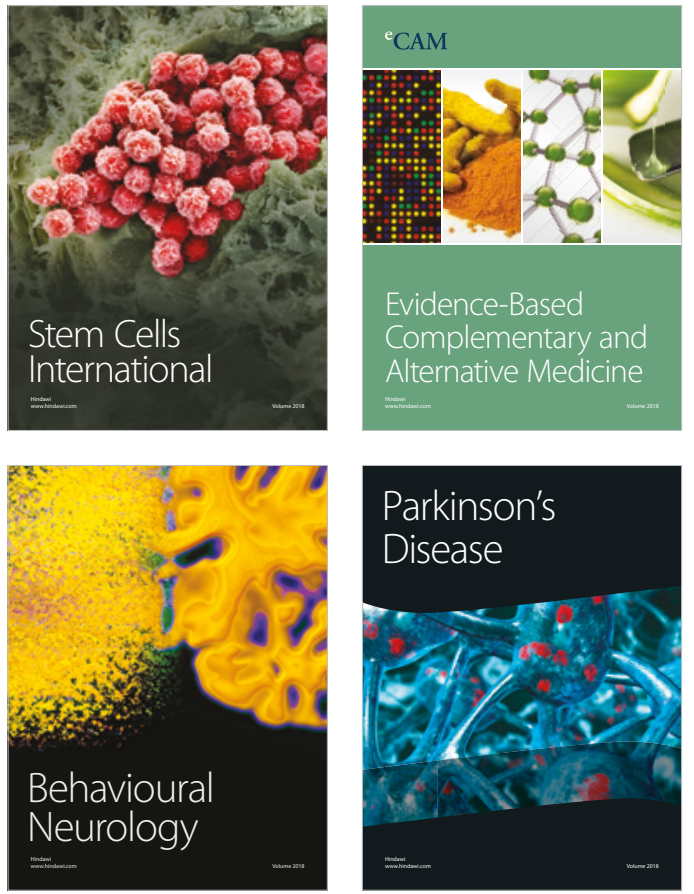

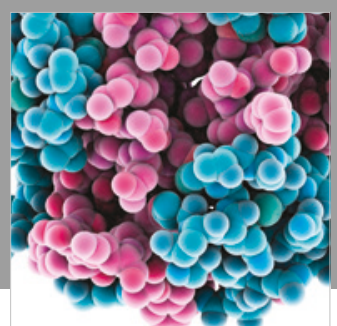

ournal of

Diabetes Research

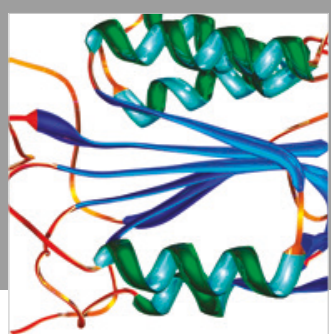

Disease Markers
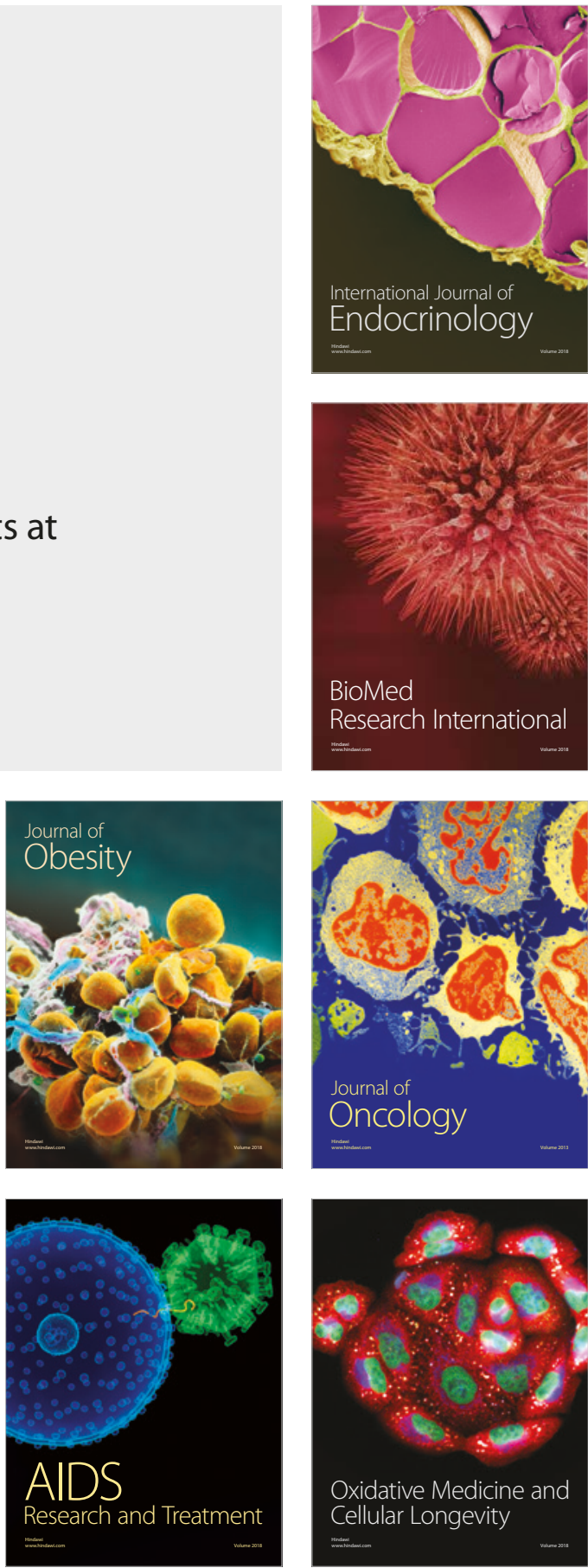